\title{
Leadless Pacing: Current State and Future Direction
}

\author{
Matthias Merkel · Philipp Grotherr · Andrea Radzewitz · Claus Schmitt
}

Received: May 12, 2017 / Published online: July 13, 2017

(C) The Author(s) 2017. This article is an open access publication

\section{ABSTRACT}

Leadless pacing is now an established alternative to conventional pacing with subcutaneous pocket and transvenous lead for patients with class I or II single-chamber pacing indication. Available 12-month follow-up data shows a $48 \%$ fewer major complication rate in patients with Micra ${ }^{\mathrm{TM}}$ compared to a historical control group in a nonrandomized study [1]. There is one system with Food and Drug Administration (FDA) approval and two with the Communaute Européenne (CE) mark. The OPS code for the implantation is $8-83 \mathrm{~d} .3$ and the procedure has recently been rated as a "new Examination and Treatment Method (NUB)" in the German DRG system, meaning adequate reimbursement is negotiable with health insurance providers. The systems offer similar generator longevity and programming possibilities as conventional pacemaker systems, including rate response, remote monitoring, and MRI safety. The biggest downsides to date are limitations to

Enhanced content To view enhanced content for this article go to http://www.medengine.com/Redeem/ OED8F06008331DBE.

M. Merkel ( $₫)$ · P. Grotherr · A. Radzewitz . C. Schmitt

Medizinische Klinik IV, Städtisches Klinikum

Karlsruhe, Moltkestrasse 90, 76133 Karlsruhe, Germany

e-mail: matthias.merkel@klinikum-karlsruhe.de single-chamber stimulation, lack of long-time data, and concerns of handling of the system at the end of its life span. However, implant procedure complication rates and procedure times do not exceed conventional pacemaker operations, and proper training and patient selection is provided.

Keywords: Complication rates of pacemaker implantation procedures; Conventional pacemakers; Leadless pacing

\section{INTRODUCTION}

Pacing is the only treatment option for permanent bradycardia. While current generator programming possibilities are close to perfect, the transvenous lead(s) and the generator pocket remain the Achilles' heel of the system. Two- to 6-month complication rates of $9.5 \%-12.4 \%$ in nationwide cohort studies have been reported $[2,3]$. Advances in battery technology and "deep miniaturization" of electronics, (i.e. 90\% system size reduction) now offer the opportunity to implant the whole system into the right ventricle (RV). This enables an alternative way of VVI-R stimulation, which, of course, currently limits the possible recipients to class I or II indication single-chamber pacing mode patients. In 2017, two devices, the Nanostim ${ }^{\mathrm{TM}}$ leadless cardiac pacemaker (LCP, St. Jude 
Medical) and the Micra ${ }^{\mathrm{TM}}$ transcatheter pacing system (TPS, Medtronic) were CE-approved. Additionally, the Micra ${ }^{\mathrm{TM}}$ has FDA approval. A third device, also tine shaped, is currently being developed, intended for release in 2018 by Boston Scientific and will be able to communicate with the company's subcutaneous defibrillator. However, the system retail prices of leadless pacemakers are about tenfold higher than those of conventional single-chamber pacemakers.

\section{COMPARISON OF THE TWO SYSTEMS}

The two available systems are both delivered via the femoral vein by a deflectable catheter/ sheath system to the right ventricle, but the LCP is screwed into the myocardium by its apical helix (depth: max. $1.3 \mathrm{~mm}$ ) while the TPS is fixated by four nitinol tines, the sharp ends facing inward to the cavum once deployed. Both weigh $2 \mathrm{~g}$, but the Nanostim ${ }^{\mathrm{TM}}$ is longer $(41.4 \mathrm{~mm})$ and narrower. Therefore, it can be implanted through an $18 \mathrm{~F}$ sheath, while the Micra ${ }^{\mathrm{TM}}$ is $25.9 \mathrm{~mm}$ long and requires a $23 \mathrm{~F}$ sheath $(27 \mathrm{~F}$ outer diameter). The preferred implantation site is the RV septum, though apical deployment is also acceptable. The RV free wall should be avoided, in order to prevent perforations. Both systems allow response rate programming via temperature sensor (LCP) or accelerometer (TPS) and both are MRI conditional at 1.5 Tesla (TPS also at 3 Tesla). The intracardiac devices work at $0.24 \mathrm{~ms}$ standard output time (TPS) or $0.4 \mathrm{~ms}$ (LCP), allowing for a battery longevity of 14.7 years (LCP) compared to 10 years (TPS), when International Organization for Standardization (ISO) conditions (i.e. $1.5 \mathrm{~V}$ output at $600 \mathrm{ohms}, 60 / \mathrm{min}, 100 \%$ stimulation) are applied. Both systems are equipped with a proximal groove to allow potential extraction by a snare. Micra ${ }^{\mathrm{TM}}$ has the ability of remote monitoring via Medtronic Carelink ${ }^{\mathrm{TM}}$ system but Nanostim ${ }^{\mathrm{TM}}$ is not linkable to the corresponding St Jude Merlin net ${ }^{\mathrm{TM}}$.

\section{CLINICAL DATA/LITERATURE REVIEW}

Nanostim LCP: The LEADLESS clinical evaluation enrolled 33 subjects, 32 of whom could be successfully implanted; one patient that developed cardiac perforation during the implantation consequently received emergency cardiac surgery and died 5 days later due to a stroke. A second device was unintentionally implanted into the left ventricle through an open foramen ovale, but could be successfully retrieved and corrected by a second device. The 3-month complication-free rate was $94 \%$ [4].

The following LEADLESS II clinical trial evaluated the LCP in a nonrandomized study in 527 subjects [5]. Implantation success was $95.8 \%$, major adverse events occurred in $6.5 \%$, including cardiac perforation in 8 patients, acute device dislodgement in 6 , and device retrieval due to elevated pacing thresholds in 4 patients. All migrated devices could be successfully retrieved percutaneously, four from the pulmonary artery and two from the femoral vein. Vascular access complication rate was $1.2 \%$. The complication rate tended to drop by half after the first 10 implant procedures.

The perforation risk issue led to the first voluntary temporary halt of Nanostim implantations, according to data in a European "Dear Doctor" letter dated Feb. 20, 2015. After a mandatory extended phased training program for implanting physicians and an update of instructions for use, including addition of specific warnings and emphasis on key implantation steps, the pericardial effusion/ perforation risk dropped from $4.1 \%$ in the 147 patients of EU pre-pause registry to $1.5 \%$ in the Leadless II study $(n=526)$ [6]. A second temporary halt of new LCP implantation was announced on Oct 26, 2016 by the German Medical Device Approval Authority BfArM after seven devices of 1.423 implanted LCPs $(0.50 \%)$ could no longer be interrogated due to accelerated battery depletion [7].

Medtronic Micra TPS: The Micra TPS investigational device study enrolled 725 subjects in a multicentre, prospectively, nonrandomized manner [8]. The evaluation compared against a 
historical cohort of six historic Medtronic conventional transvenous pacing system evaluations. The analysis began when 300 patients reached 6 months of follow-up. In 719 of 725 subjects $(99.2 \%)$, the system was successfully implanted. The primary safety endpoint was $96 \%$, thus reaching a complication rate of $4 \%$ and the efficacy endpoint was $98.3 \%$. Among the complications was one death due to metabolic acidosis (underlying renal failure, possibly sepsis), 11 cardiac effusions, 5 groin puncture complications, but no dislodgement or confirmed systemic infection.

The comparison to the historical conventional pacing group $(n=2667)$, revealed through 6 months of follow-up, showed that the research cohort experienced fewer major complications than in the historical control cohort $(4.0 \%$ vs. $7.4 \%$; hazard ratio $0.49 ; 95 \%$ CI $0.33-0.75 ; P=0.001)$.

Duray, Ritter, el-Charmi et al. recently published 1-year results of the above-mentioned Micra Transcatheter Pacing Study Group patients [1]. The complication-free rate remained stable at $96 \%$, with 4 new complications since the primary results' analysis: 3 new heart failures and 1 pacemaker syndrome. No device dislodgements, telemetry failures, or device infections occurred.

This adds to a likewise constant reduction of complications in comparison to the same historical control group of conventional pacemaker recipients of $48 \%$ (HR 0.52 , CI $0.35-0.77$ ).

\section{COMPARISON MICRA ${ }^{T M}$ TO NANOSTIM ${ }^{\text {TM }}$}

To date, there are no direct comparisons between the two currently available leadless pacing devices, though their implant complication rates and 6-month performance seem comparable, apart from acute device dislodgement, which is so far only reported in Nanostim recipients (at a rate of $1.1 \%$ in Leadless II [5]).

\section{Comparison of Leadless Pacemakers to Traditional Pacemaker Systems}

The reported complication rates of leadless pacemaker implantation trials of $4 \%-6.5 \%$ compare favourably to available transvenous data.

In the Micra TPS trial [1], the historical control group of 2667 de novo (of note: singleand DUAL-chamber) pacemaker recipients experienced a total major complication rate of $7.6 \%$ compared to $4.0 \%$ in the leadless group after 12 months (hazard ratio 0.52 , 95\% CI $0.35-0.77 ; P=0.001)$. The reduction in major complication rate was associated with a $47 \%$ reduction in prolonged hospitalisation and $82 \%$ reduction rate in system revisions.

Nationwide analyses of cardiac pacemaker implantation complications are available for Denmark by Kirkfeldt et al. [3]. They report a complication rate of $7.5 \%$ in single lead system implants and a $12.5 \%$ rate with dual lead transvenous pacing systems. The Follow Pace Study reported a similar rate of complications $(12.5 \%)$ after single and dual chamber de novo implants [2].

The rate of cardiac perforation/pericardial effusion is greater in leadless pacemaker implantation than in transvenous systems. A meta-analysis of 28 studies comprising 60,744 patients found a mean incidence of lead perforation of $0.82 \%$ [9], whereas the rate of cardiac perforation/pericardial effusion in the two leadless PM studies was $1.6 \%$ for each.

Patients especially suitable for leadless pacing: obviously, leadless pacing is the only pacing possibility (apart from epimyocardial access with its intrinsic difficulties to provide low and stable thresholds) when the upper central venous system is damaged. This can occur after previous transvenous pacemaker or indwelling catheter infections, chest surgery, radiation for thoracic tumors, and trauma, among other reasons.

Leadless pacing should be strongly considered in patients on haemodialysis for two reasons: Firstly, for sparing the upper venous system, meaning also the access for dialysis, and, secondly, their increased rate of transient bacteriemia while being dialysed leading to haematogenous lead infection. So far, pacemaker infections have not been reported in leadless systems, presumably due to high blood flow velocities, small device size, and encapsulation in the heart. 
A point of discussion is whether young adults with neurocardiogenic syncopes of the cardioinhibitory type should be offered a leadless pacing system (Fig. 1 and videos 1, 2, 3, 4 are available in enhanced content-med engine link). Of course, pacing indications in these patients should have been chosen very prudently, but on the other hand we all know the occasional young woman with frequent syncopes and documented asystoles in the range of 30 s leads to social isolation over time. We think it is justified to offer the leadless system as an alternative to conventional indwelling pacing leads given the risks of carrying them over decades to come. Even when abandoned later, they remain a time bomb ready to infect, break, or migrate at any time. Anybody who ever laser-extracted completely ingrown calcified pacemaker leads, placed in the venous system for decades, knows about the downsides. Last but not least, there is a cosmetic advantage especially taken seriously by young ladies (this sometimes results in the forgetting of being a pacemaker patient in carriers of leadless systems, since nothing reminds the patient of it).
Patients for whom leadless pacing is considered face enhanced risks. The trials identified the following risk factors for implant complications (i.e. perforation risk): elderly, frail women, low body mass index, patients on chronic steroids (this applies also for transvenous system implantations). In our own experience, the implantation can be technically challenging when dealing with enlarged right atrial and/or right ventricular diameters, generally in pulmonary hypertension patients. The preformed and fixed bending radius of the delivery sheath makes it more difficult to reach the RV and to deploy the system at the desired point and, therefore, it is prudent to avoid these patients when beginning with the technique.

\section{OWN RESULTS}

In our own series of the first 25 leadless pacemaker (Micra ${ }^{\mathrm{TM}}$ ) recipients (15 male, 10 female, age $25-89$ years), the average procedure time was $29 \mathrm{~min}$ and the fluoroscopy time was $6.7 \mathrm{~min}$. On average, we needed one repositioning manoeuvre to achieve an acute pacing

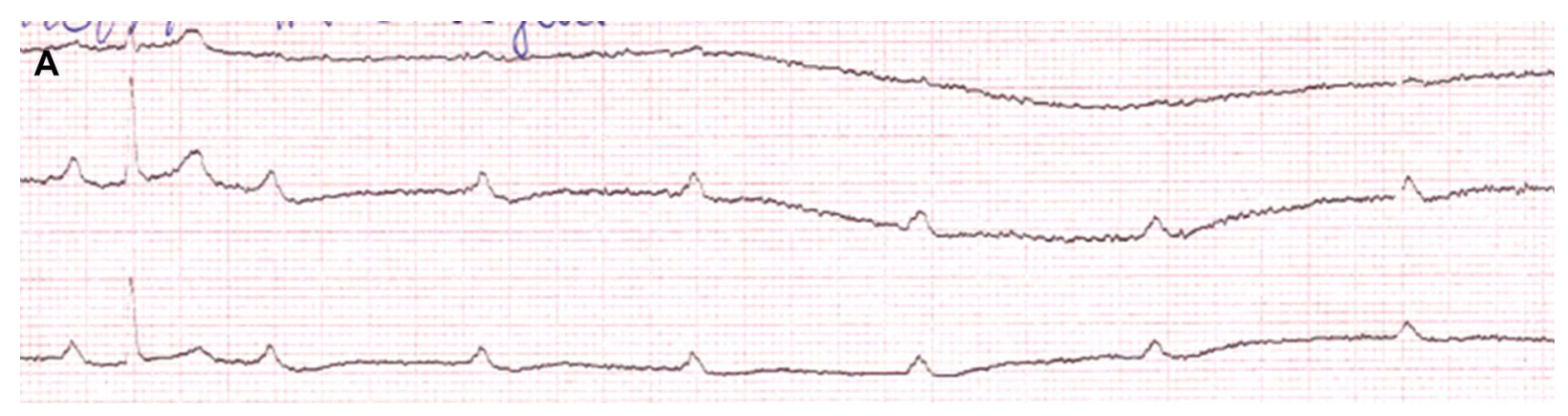

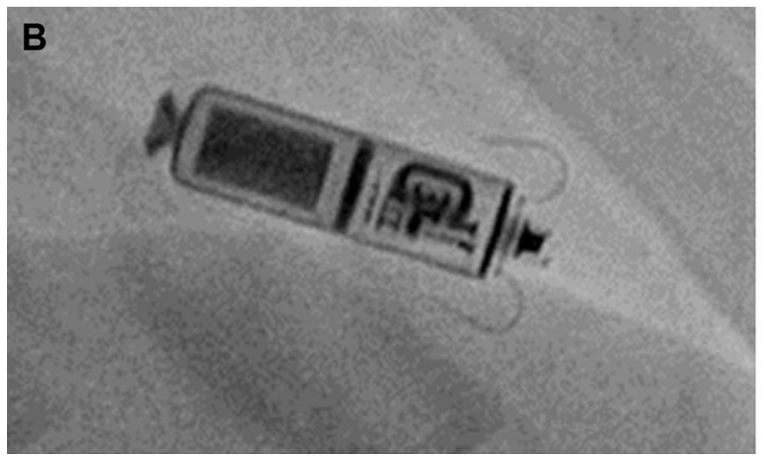

Fig. 1 24-year-old female with recurrent syncope. a Tilt table test: after $4 \mathrm{~min}$ in the upright position complete AV-block with syncope. $\mathbf{b}$ Implantation of a leadless pacer
$\left(\mathrm{Micra}^{\mathrm{TM}}\right)$ in the septal region of the right ventricle (see videos 1, 2, 3, 4 are available in enhanced content-med engine link) 
threshold of $0.48 \mathrm{~V} @ 0.24 \mathrm{~ms}$ and sensing of $11.9 \mathrm{mV}$, respectively. No serious adverse event occurred during implantation or during the observation period so far. There was only one conservatively treated groin complication (AV fistula) and no device dislodgement, cardiac complication, or procedural death in our series. Of note, 21 patients were implanted under continued anticoagulation: 10 on phenprocoumon, 9 on novel oral anticoagulants (NOAK), and 2 on intravenous heparin. Amongst these 21 anticoagulated patients, 3 patients were treated with combined anticoagulation with clopidogrel and one patient was under triple-therapy with ASS, clopidogrel, and phenprocoumon. (Of note: the companies leave periprocedural anticoagulation to the discretion of the implanting physician; it is neither recommended nor discouraged). Two patients were on hemodialysis, one without upper venous access after bilateral neck dissection and radiotherapy for squamous cell carcinoma of the mouth. Two patients presented with infected transvenous pacing systems (CRT-D on the one hand and dual-chamber pacemaker on the other hand). Both were not pacemaker-dependent and had their whole system extracted and systemic infection successfully treated ( 2 months and 7 days, respectively) before the leadless implantation.

To date, all systems are working without technical complications. The pacing threshold, sensing, and impedance so far remained stable during the first observational year: pacing threshold0.88 V@0.24 ms, sensing 12.2 V (9/25 patients controlled at 1 year follow-up so far). Two patients experienced a worsening threshold of 2.5 V@0.24 ms and 2.1 V@0.24 ms, respectively, at the 6-month visit which was tolerated due to very old age in one patient and concomitant active cancer in the other. All remaining patients so far stayed at thresholds under $1 \mathrm{~V}$, thus offering a projected device longevity of $>10$ years. Compliance with Ethics Guidelines: This article does not contain any new studies with human or animal subjects performed by any of the authors.

Which pacing threshold at implantation is acceptable? Piccini et al. recently examined the long-term outcomes of the Micra ${ }^{\mathrm{TM}}$ pacemakers in the TPS study population with elevated thresholds at implantation [10]. Of the 711 patients with threshold data at implantation, 83 (11.7\%) had a threshold of $>1.0 \mathrm{~V}$ at $0.24 \mathrm{~ms}$. The threshold decreased significantly during the first 6 months with $85 \%$ having 6-month thresholds lower than the implant value. However, when the capture threshold at implantation was $>2 \mathrm{~V}$, only $18.2 \%$ had a threshold of $\leq 1 \mathrm{~V}$ at 6 months and $45.5 \%$ had a capture threshold of $>2 \mathrm{~V}$ [10]. The authors conclude that aggressive repositioning of the device may not be necessary as long as the threshold is below2 V@0.24 ms. However, at values $>2 \mathrm{~V}$, repositioning is strongly recommended to ensure stable capture of the myocardium.

\section{UNANSWERED QUESTIONS}

The obvious limitation to RV single-chamber pacing is the biggest concern to date. Communicating systems are currently being developed. The first partner device will probably be the subcutaneous defibrillator in the upcoming Boston Scientific Marlborough, Ma leadless pacemaker (intended release 2018). It will offer both antibradycardia and antitachycardia (ATP) pacing opportunities. Furthermore, by combining intracardiac signals acquired by the leadless pacer and the surface ECGs processed by the S-ICD may hopefully offer new and unique possibilities to eliminate inadequate ICD shocks, e.g. in SVT, tachyarrhythmia, or T wave over-sensing.

Since the majority of pacemaker patients depend on AV synchronization, or even additional cardiac resynchronization, i.e. dual ventricular chamber pacing, the breakthrough of the leadless technology will only be possible after a solution for atrial sensing by the system is found. Companies are already searching for a way of preserving $\mathrm{AV}$-sequential stimulation, probably by sensing the $P$ wave and via $\mathrm{VDD}(\mathrm{R})$ stimulation mode. One can speculate that this might be possible by sampling a surface-ECG-like recording. Currently available implantable loop recorders, for example, are already capable of a reliable $\mathrm{P}$ wave detection.

The second biggest concern is generator handling at the end of the system's life span. 
Unlike in conventional systems, battery depletion means complete system extraction of the leadless system. Companies are developing extraction tools and both systems are equipped with extraction grooves to be grabbed by snares. The systems seem to be safely retrievable in the acute phase with snares, potentially facilitated by deflectable electrophysiology catheters and/ or sheaths.

However, data of extraction of chronically implanted devices are limited, and there is no experience with extraction of systems implanted for longer than 3 years in humans. Reddy et al. report successful extraction of 10/11 devices implanted $>6$ weeks (range 88-1188 days) [11] and Bongiorni et al. and Bonner et al. after 28 months in sheep [12, 13]. Of note, the devices seem to have the potential to become completely encapsulated into the heart over time. In this scenario, they can be programmed off (OOO mode) and up to three devices can be accommodated in the human right ventricle [14]. This adds to a potential pacing time of 30-45 years with sequential leadless pacing, given good thresholds. However, since the average recipient is $70-80$ years old, one device should suffice for the majority of patients.

Until the extraction issue is resolved, we have to rely on the possibility of abandoning the system and programming them "off" (000 mode). This has to lead to a strategy of managing patients with sequential leadless pacemaker devices over a long interval. The occupied volume of $1 \mathrm{ml}$ should not compromise an adult RV. Uncertainty about safety to bloodstream infection, system migration, and $\mathrm{RV}$ failure in the very long run remains though.

\section{CONCLUSION}

The leadless pacing technology is a promising new and viable option for permanent cardiac pacing with excellent clinical data and the potential of halving the complication rate in comparison to the established systems. Its limitation to VVI-R pacing and unsolved issues about long-term extractability indicate that we currently are only at the beginning of a new era which will undoubtedly address pacemaker lead and pocket complications. Leadless pacers enable placing the system where it belongs to, namely inside the heart.

As Mela and Singh put it: "The leadless technology has made it into clinical practice and is here to stay" [15].

\section{ACKNOWLEDGEMENTS}

No funding or sponsorship was received for this study or publication of this article. All named authors meet the International Committee of Medical Journal Editors (ICMJE) criteria for authorship for this manuscript, take responsibility for the integrity of the work as a whole, and have given final approval for the version to be published. All authors had full access to all of the data in this study and take complete responsibility for the integrity of the data and accuracy of the data analysis.

Disclosure. Matthias Merkel is consultant and has received a speakers honorarium from Boston Scientific and Medtronic. Claus Schmitt received speakers honorarium from Boston Scientific and Medtronic and financial support of scientific meetings. Philipp Grotherr has received a speakers honorarium from Boston Scientific and Medtronic. Andrea Radzewitz declares no conflict of interest.

Compliance with Ethics Guidelines. This article does not contain any new studies with human or animal subjects performed by any of the authors.

Data Availability. The datasets analysed during the current study are available from the corresponding author on reasonable request.

Open Access. This article is distributed under the terms of the Creative Commons Attribution-NonCommercial 4.0 International License (http://creativecommons.org/licenses/ by-nc/4.0/), which permits any noncommercial use, distribution, and reproduction in any medium, provided you give appropriate credit 
to the original author(s) and the source, provide a link to the Creative Commons license, and indicate if changes were made.

\section{REFERENCES}

1. Duray GZ, Ritter P, El-Charmi M, et al. Long-term performance of a transcatheter pacing system: 12-month results from the Micra transcatheter Pacing Study. Heart Rhythm. 2017;14:702-9.

2. Eo U, Zuithoff NP, van Hemel NM, de Cock CC, Hendriks T, Doevendans PA, Moons KG. Incidence and predictors of short- and long-term complications in pacemaker therapy: the FOLLOWPACE study. Heart Rhythm. 2012;9:728-35.

3. Kirkfeldt RE, Johansen JB, Nohr EA, Jørgensen OD, Nielsen JC. Complications after cardiac implantable electronic device implantations: an analysis of a complete, nationwide cohort in Denmark. Eur Heart J. 2014;35:1186-94.

4. Reddy VY, Knops RE, Sperzel J, et al. Permanent leadless cardiac pacing: results of the LEADLESS trial. Circulation. 2014;129:1466e-71e.

5. Reddy VY, Exner DV, Cantillon DJ, et al. Percutaneous implantation of an entirely intracardiac leadless pacemaker. $\mathrm{N}$ Engl J Med. 2015;373:1125-35.

6. St. Jude Medical Nanostim Leadless Pacemaker Panel Pack for Circulatory System Devices Panel Meeting Date: February 18, 2016. https://www.fda. gov/downloads/AdvisoryCommittees/Committees MeetingMaterials/MedicalDevices/MedicalDevices AdvisoryCommittee/CirculatorySystemDevicesPanel/ UCM485095.pdf. Accessed 20 January 2016.
7. BfArM Customer Information. http://www.bfarm. de/SharedDocs/Kundeninfos/DE/01/2016/08980_ 16_Kundeninfo_de.pdf?_blob=publicationFile\&v= 2. Accessed 28 Oct 2016.

8. Reynolds D, Duray ZG, Omar R, et al. A leadless intracardiac transcatheter pacing system. N Engl J Med. 2015;. doi:10.1056/NEJMoa1511643.

9. Vamos M, Erath JW, Benz AP, Bari Z, Duray GZ, Hohnloser SH. Incidence of cardiac perforation with conventional and with leadless pacemaker systems: a systematic review and meta-analysis. J cardiovasc Electrophyiol. 2017;28:336-46.

10. Piccini JP, Stromberg K, Jackson KP, et al. Long-term outcomes in leadless Micra transcatheter pacemakers with elevated thresholds at implantation: results from the Micra Transcatheter Pacing System Global Clinical Trial. Heart Rhythm. 2017;14:685-91.

11. Reddy VY, Miller MA, Knops RE, et al. Retrieval of the leadless cardiac pacemaker: a multicenter experience. Circ Arrhythm Electrophysiol. 2016;9:e004626.

12. Bongiorni MG, Segreti L, Di Cori A, Bonner M, Eggen M, Omdahl P. Retrieval of a transcatheter pacemaker in sheep after a mid-term implantation time. Heart Rhythm Case Rep. 2016;2:43-6.

13. Bonner MD, et al. Extraction of the Micra transcatheter pacemaker System. Presented at HRS 2014. Poster Session IV.

14. Omdahl P, Eggen MD, Bonner MD, Iaizzo PA, Wika K. Right ventricular anatomy can accommodate multiple transcatheter pacemakers. Pacing Clin Electrophysiol. 2016;39:393-7.

15. Mela T, Singh JP. Leadless pacemakers: leading us into the future? Eur Heart J. 2015;1(36):2520-2. 